\title{
Deformations of topological spaces predicted by $E$-theory
}

\author{
Marius Dădărlat * \\ Department of Mathematics \\ University of Maryland \\ College Park, MD 20742
}

\author{
Terry A. Loring ${ }^{\dagger}$ \\ Department of Mathematics and Statistics \\ University of New Mexico \\ Albuquerque, NM 87131
}

\section{Introduction}

Let $X$ be a locally compact space. By a deformation of $X$ we mean a continuous field $\left\{A_{t} \mid t \in[0,1]\right\}$ of $C^{*}$-algebras with $A_{0} \cong C_{0}(X)$, and

$$
\left\{A_{t} \mid t \in(0,1]\right\} \cong B \times(0,1]
$$

for a fixed $C^{*}$-algebra $B$. Replacing $C_{0}(X)$ by another $C^{*}$-algebra $A$, we generalize this to a deformation of one $C^{*}$-algebra to another. This is a basic interpretation of deformation-it reflects only the topology of $X$ and omits more general fields of algebras - but is an important one. This importance is seen in the relation to $E$-theory and the examples $[3,8,11,12]$ that have arisen.

Deformations are, in fact, very common. About the only requirement for a $C^{*}$-algebra to arise as a deformed $\mathrm{CW}$-complex is that it have the correct $K$-theory. This fact follows from our calculations in "unsuspended" $E$-theory [5]. We will explicitly describe one of the deformations predicted by these calculations: a deformation of a three-dimensional CW complex into a dimension-drop interval. We hope this example will further clarify the role of the dimension-drop interval as a building block in Elliott's inductive limits [6].

${ }^{*}$ current address: Department of Mathematics, Purdue University, West Lafayette, IN 47907

†partially supported by NSF grant DMS-9007347 
Recall, from [3], that an asymptotic morphism $\left(\varphi_{t}\right): A \rightarrow B$ between $C^{*}$-algebras is a collection of maps $\varphi_{t}: A \rightarrow B$ for $t \in[1, \infty)$ such that for $a, b \in A$ and $\alpha \in \mathbf{C}$, as $t \rightarrow \infty$,

$$
\begin{aligned}
\left\|\varphi_{t}(a b)-\varphi_{t}(a) \varphi_{t}(b)\right\| & \rightarrow 0, \\
\left\|\varphi_{t}\left(a^{*}\right)-\varphi_{t}(a)^{*}\right\| & \rightarrow 0, \\
\left\|\varphi_{t}(\alpha a+b)-\alpha \varphi_{t}(a)-\varphi_{t}(b)\right\| & \rightarrow 0
\end{aligned}
$$

and $t \mapsto \varphi_{t}(a)$ is continuous. We say that $\left(\varphi_{t}\right)$ is injective if also lim sup $\left\|\varphi_{t}(a)\right\|>0$ for all $a$.

Injective asymptotic morphisms correspond exactly to deformations. Thus, we will work in the context of asymptotic morphisms. See [3] for an explanation of this correspondence and the definitions of equivalence and homotopy for asymptotic morphisms.

The following result often gives the easiest way to show a given asymptotic morphism is injective. First, we recall how an asymptotic morphism $\left(\varphi_{t}\right): A \rightarrow B$ induces maps on K-theory. Given a projection $p$ in $A$, the class of $\varphi_{*}([p])$ in $K_{0}(B)$ is represented by any projection that is close to $\varphi_{t}(p)$ for some sufficiently large value of $t$. For projections, and unitaries, in $M_{k}(A)$ a similar construction is used.

Proposition 1 Suppose $X \cup\{p t\}$ is a compact manifold. If an asymptotic morphism $\left(\varphi_{t}\right)$ : $C_{0}(X) \rightarrow B$ induces an injective map on $K$-theory then $\left(\varphi_{t}\right)$ is injective.

Remark 2 This type of result holds more generally. In particular, it holds for the CW complex discussed in section 3 .

Example 3 Our first exampe is an asymptotic morphism $\left(\alpha_{t}\right): C_{0}\left(\mathbf{R}^{2}\right) \rightarrow \mathcal{K}$ which induces an isomorphism on $K$-theory. This well-known in several contexts.

We regard $C_{0}\left(\mathbf{R}^{2}\right)$ as the universal $C^{*}$-algebra generated by selfadjoint element $h$ and normal element $N$ subject to the relation $h=h^{2}+N^{*} N$. so that the generator of $K_{0}\left(C_{0}\left(\mathbf{R}^{2}\right)\right) \cong \mathbf{Z}$ is just

$$
\left[\left(\begin{array}{cc}
h & N^{*} \\
N & 1-h
\end{array}\right)\right]-\left[\left(\begin{array}{ll}
1 & 0 \\
0 & 0
\end{array}\right)\right] .
$$

An asymptotic morphism from $A$ to $B$ is given, up to equivalence, by a $*$-homomorphism from $A$ to $B_{\infty}$, where $B_{\infty}$ is the $C^{*}$-algebra described in [3]. Therefore, if $A$ is universal 
for a set of relations one need only define the paths in $B$ that are to be the images of the generators. In this case, we need only define $\alpha_{t}(h)$ and $\alpha_{t}(N)$.

Let $S$ denote the unilateral shift and, for $t \in[1, \infty)$, let $D_{t}$ denote the diagonal operator whose diagonal corresponds to the sequence

$$
1 / t, 2 / t, \ldots,[t] / t, 1,1, \ldots
$$

Set $\alpha_{t}(h)=1-D_{t}$ and $\alpha_{t}(N)=\sqrt{D_{t}-D_{t}^{2}} S$. Since the required relations hold asymptotically, this determines $\alpha_{t}$. The fact that this induces an isomorphism on $K_{0}$ follows from the calculation, in [2] or [7], of the spectrum of

$$
\left[\begin{array}{cc}
1-D_{t} & \sqrt{D_{t}-D_{t}^{2}} S^{*} \\
\sqrt{D_{t}-D_{t}^{2}} S & D_{t}
\end{array}\right] .
$$

See [9] for more details and a modification of this example that produces deformations of $\mathbf{R P}^{2}$ and the Klein bottle.

\section{Unsuspended E-theory}

Let $A$ and $B$ be $C^{*}$-algebras. For convenience, we shall assume that $A$ and $B$ are separable and nuclear. We will use the notation

$$
\begin{aligned}
{[A, B] } & =\text { homotopy classes of } * \text {-homomorphisms, } \\
{[[A, B]] } & =\text { homotopy classes of asymptotic morphisms. }
\end{aligned}
$$

We will use the following isomorphisms, from [3],

$$
\begin{aligned}
K K(A, B) & \cong E(A, B) \\
& \cong[[S A \otimes \mathcal{K}, S B \otimes \mathcal{K}]] \\
& \cong\left[\left[S^{2} A \otimes \mathcal{K}, S^{2} B \otimes \mathcal{K}\right]\right]
\end{aligned}
$$

We now arrive at our main result. By $X \cup\{\mathrm{pt}\}$ we mean the one-point compactification of a locally compact space $X$. Combined with Proposition 1 this result guarantees the existence of many deformations. 
Proposition 4 If $X \cup\{p t\}$ is a connected, finite $C W$ complex then the suspension map

$$
\left[\left[C_{0}(X), B \otimes \mathcal{K}\right]\right] \rightarrow K K\left(C_{0}(X), B\right)
$$

is an isomorphism.

The proof of this will be given in [5]. The inverse map may be described as follows. Let

$$
\beta: C_{0}\left(\mathbf{R}^{1}\right) \rightarrow C_{0}\left(\mathbf{R}^{3}\right) \otimes \mathcal{K}
$$

be a $*$-homomorphism inducing an isomorphism on $K$-theory. By [4, Corollary 3.1.8] there exists a map

$$
\beta_{X}: C_{0}(X) \rightarrow S^{2} C_{0}(X) \otimes \mathcal{K}
$$

whose suspension is homotopic to $\beta \otimes \operatorname{id}_{C_{0}(X)}$. Composition, on appropriate sides, by $\beta_{X}$ and the asymptotic morphism

$$
1 \otimes \alpha: S^{2} B \otimes \mathcal{K} \rightarrow B \otimes \mathcal{K}
$$

(see Example 3) defines the inverse mapping

$$
\left[\left[S^{2} C_{0}(X) \otimes \mathcal{K}, S^{2} B \otimes \mathcal{K}\right]\right] \rightarrow\left[\left[C_{0}(X), B \otimes \mathcal{K}\right]\right]
$$

Using the universal coefficient theorem we obtain a corollary.

Corollary 5 If $X \cup\{p t\}$ is a finite $C W$ complex and $\eta: K^{*}(X) \rightarrow K_{*}(B)$ is an isomorphism then there exists a deformation of $X$ to $B \otimes \mathcal{K}$ which induces $\eta$.

\section{Matricial torsion}

Consider the three-dimensional CW complex obtained by attaching, with degree two, the boundary of a three-cell $B^{3}$ to a two-sphere $S^{2}$. Remove the base-point (which sits in the copy of $S^{2}$ ) and call the result $X$. That is,

$$
X \cup\{\mathrm{pt}\}=B^{3} \cup_{\zeta} S^{2}
$$

where $\zeta: \partial B^{3} \rightarrow S^{2}$ has degree 2 . Thus $K_{0}\left(C_{0}(X)\right)=0$ and $K_{1}\left(C_{0}(X)\right) \cong \mathbf{Z} / 2$. 
Let $B$ denote the non-unital dimension-drop interval, that is,

$$
B=\left\{f \in C_{0}\left((0,1], M_{2}\right) \mid f(1) \text { is scalar }\right\}
$$

One may compute $K_{0}(B)=0$ and $K_{1}(B) \cong \mathbf{Z} / 2$.

We know, by Corollary 5 , that there is an asymptotic morphism

$$
\left(\psi_{t}\right): C_{0}(X) \rightarrow B \otimes \mathcal{K}
$$

inducing an isomorphism on $K$-theory. This is an example of topological torsion being "quantized" into matricial torsion. Our goal is to find $\psi$ explicitly.

We first must be more explicit about the attaching map and the associated $*$-homomorphism $\theta: C_{0}\left(\mathbf{R}^{2}\right) \rightarrow C_{0}\left(\mathbf{R}^{2}\right)$. Using the generators and relations of Example 3, we determine $\theta$ by setting

$$
\begin{aligned}
\theta(h) & =f(h), \\
\theta(N) & =g_{1}(h) N+g_{2}(h) N^{*}
\end{aligned}
$$

where $f, g_{1}$ and $g_{2}$ are functions of the form

which satisfy $g_{1} g_{2}=0$ and $f(t)=f(t)^{2}+\left(g_{1}(t)^{2}+g_{2}(t)^{2}\right)\left(t-t^{2}\right)$.

We will also need $\iota: \mathcal{K} \rightarrow \mathcal{K} \otimes M_{2}$ given by $\iota(T)=T \otimes I$. With the additional notation of $\delta_{1}$ indicating evaluation at 1 , we have two pull-back diagrams:

$$
\begin{aligned}
& C_{0}(X) \quad B \otimes \mathcal{K} \\
& \begin{array}{cccc}
C_{0}(0,1] \otimes C_{0}\left(\mathbf{R}^{2}\right) & C_{0}\left(\mathbf{R}^{2}\right) & C_{0}(0,1] \otimes \mathcal{K} \otimes M_{2} & \mathcal{K} \\
\delta_{1} & \theta & \delta_{1} & \iota \\
& & & \\
& C_{0}\left(\mathbf{R}^{2}\right) & & \mathcal{K} \otimes M_{2}
\end{array}
\end{aligned}
$$


Lemma 6 There exists an asymptotic morphism $\left(\varphi_{t}\right): C_{0}\left(\mathbf{R}^{2}\right) \rightarrow M_{2}(\mathcal{K})$ such that, for all $t$,

$$
\operatorname{image}\left(\varphi_{t} \circ \theta\right) \subseteq\left\{\left[\begin{array}{ll}
T & \\
& T
\end{array}\right] \mid T \in \mathcal{K}\right\}
$$

which induces an isomorphism on $K$-theory.

We defer the proof until after we see how the lemma is used.

Consider the following commutative diagram (commuting exactly for each $t$ ):

$$
\begin{array}{ccc}
C_{0}(0,1] \otimes C_{0}\left(\mathbf{R}^{2}\right) & \text { id } \otimes \varphi_{t} & C_{0}(0,1] \otimes M_{2}(\mathcal{K}) \\
\delta_{1} & & \delta_{1} \\
C_{0}\left(\mathbf{R}^{2}\right) & \varphi_{t} & M_{2}(\mathcal{K}) \\
\theta & & \iota \\
C_{0}\left(\mathbf{R}^{2}\right) & \eta_{t} & \mathcal{K}
\end{array}
$$

Here $\eta_{t}$ is the unique solution to $\iota \circ \eta_{t}=\varphi_{t} \circ \theta$. Since $\varphi$ induces an isomorphism on $K$-theory, $\eta$ must as well.

Since these maps are not $*$-homomorphisms we cannot immediately invoke the pull-back property. However, simply restricting $\operatorname{id}_{C_{0}(0,1]} \otimes \varphi_{t}$ produces $\psi_{t}: C_{0}(X) \rightarrow B \otimes \mathcal{K}$. Now considering the $K$-theory of the commuting diagram

$$
\begin{array}{ccc}
C_{0}(0,1) \otimes C_{0}\left(\mathbf{R}^{2}\right) & \text { id } \otimes \varphi_{t} & C_{0}(0,1) \otimes M_{2}(\mathcal{K}) \\
C_{0}(X) & \psi_{t} & B \otimes \mathcal{K}
\end{array}
$$

it is easy to see that $\psi$ induces an isomorphism on $K$-theory.

Proof (Lemma 6) In order to specify $\left(\varphi_{t}\right): C_{0}\left(\mathbf{R}^{2}\right) \rightarrow M_{2}(\mathcal{K})$, it suffices to specify where the generators $h$ and $N$ are sent. At $t=n \in \mathbf{N}$, we shall have $\varphi_{n}(h)=H_{n}$ and $\varphi_{n}(N)=N_{n}$ where $H_{n}$ and $N_{n}$ are the following elements of $M_{2}\left(M_{2^{n}}\right)$ (we regard $M_{2^{n}}$ as a corner of $\mathcal{K}$ 
and so $\left.M_{2}\left(M_{2^{n}}\right) \subseteq M_{2}(\mathcal{K})\right)$ :
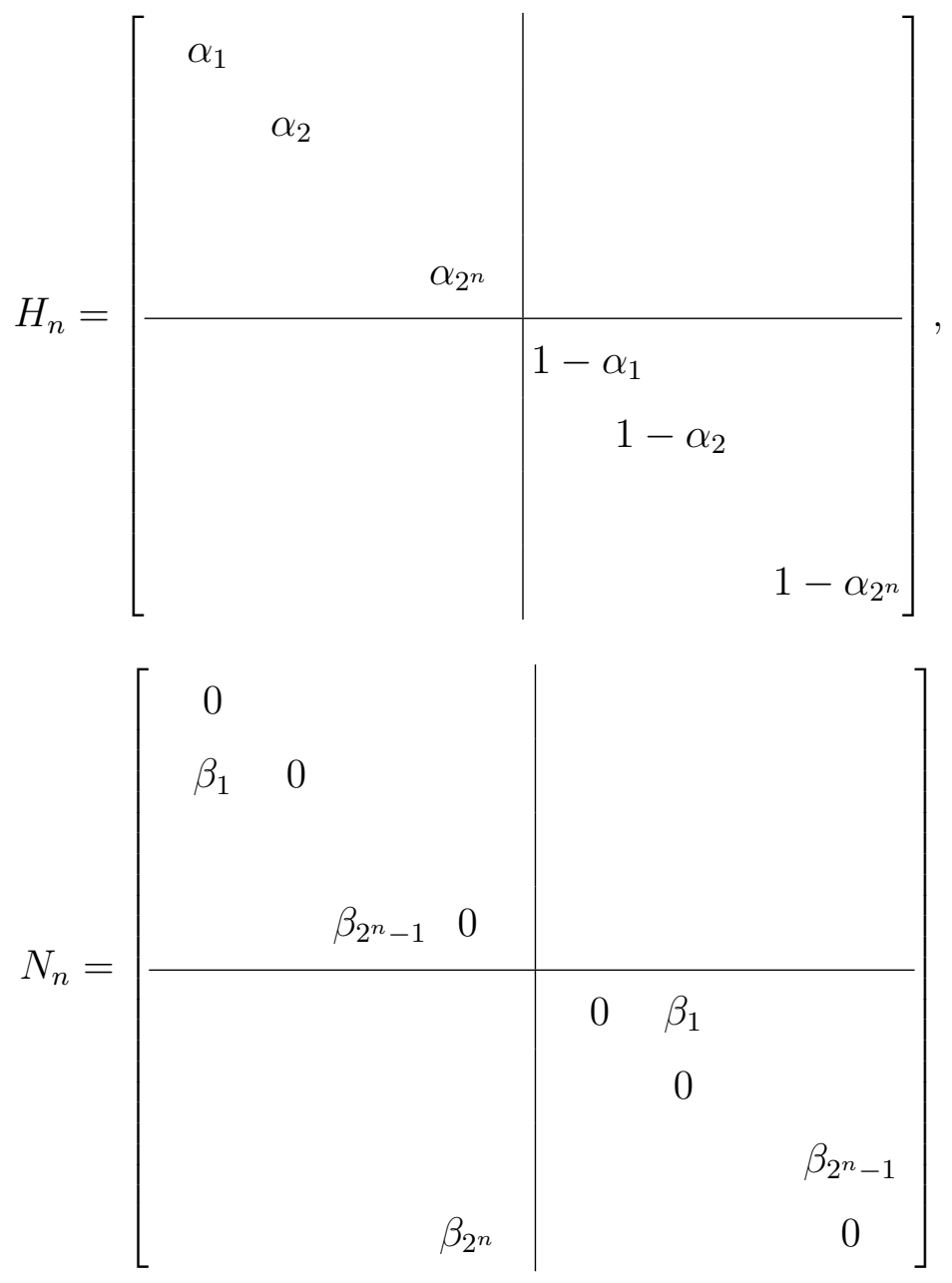

where, for $j=1, \ldots, 2^{n}, \alpha_{j}=j / 2^{n+1}$ and $\beta_{j}=\sqrt{\alpha_{j}-\alpha_{j}^{2}}$.

We are interested, more generally, in matrices $A, B \in M_{2}\left(M_{k}\right)$ such that the following relations hold:

$$
\begin{aligned}
\|[A, B]\|,\left\|\left[B, B^{*}\right]\right\|,\left\|\left[A, B^{*}\right]\right\| & \leq \epsilon \\
\left\|A-A^{*}\right\| & \leq \epsilon \\
\left\|A^{2}+B^{*} B-A\right\| & \leq \epsilon \\
f(A) & \in M_{k} \otimes I \\
g_{1}(A) B+g_{2}(A) B^{*} & \in M_{k} \otimes I
\end{aligned}
$$

It may be checked that $H_{n}$ and $N_{n}$ satisfy (1), for some $\epsilon_{n} \geq 0$, with $\epsilon_{n} \rightarrow 0$ as $n \rightarrow \infty$. 
We will need some auxiliary matrices in $M_{2}\left(M_{2^{n}}\right)$ :

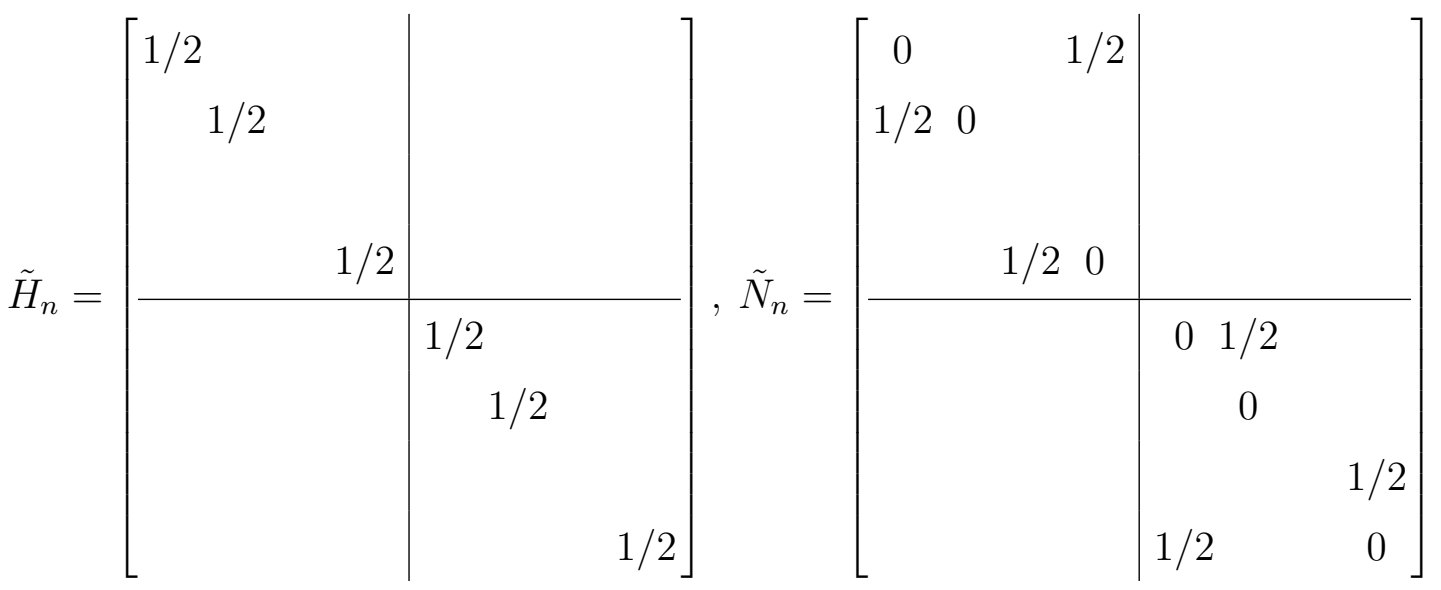

A path of unitaries, multiplied by $1 / 2$, in the lower-right-hand corner will create a path satisfying (1) from $\tilde{H}_{n}, \tilde{N}_{n}$ to

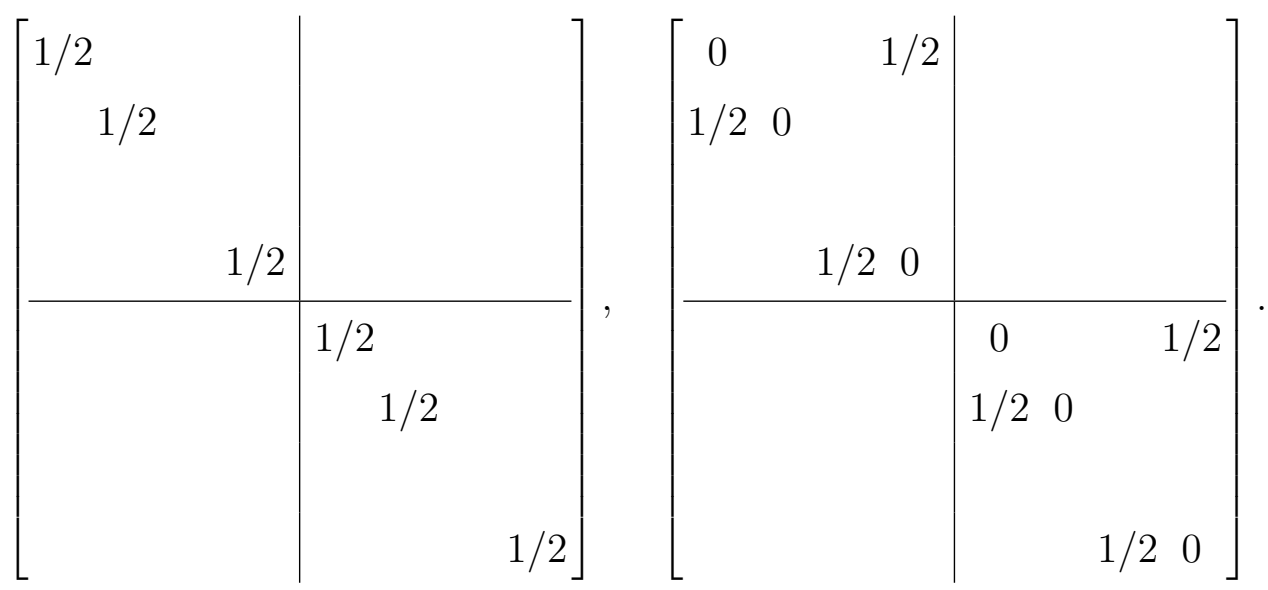

Now, deforming the pair of scalars $(1 / 2,1 / 2)$ to $(0,0)$ appropriately continues this path to the pair of matrices 0,0 . By this argument, we have reduced the construction of $\left(\varphi_{t}\right)$ to being able to connect $H_{n} \oplus \tilde{H}_{n}, N_{n} \oplus \tilde{N}_{n}$ to $H_{n+1}, N_{n+1}$ via pairs satisfying (1).

Let

$$
A_{n}=\left[\begin{array}{lll}
\alpha_{1} & & \\
& \alpha_{2} & \\
& & \\
& & \alpha_{2^{n}}
\end{array}\right], B_{n}=\left[\begin{array}{lll}
0 & & \\
\beta_{1} & 0 & \\
& & \\
& \beta_{2^{n}-1} &
\end{array}\right], C_{n}=\left[\begin{array}{lll}
0 & & \\
& 0 & \\
& & \\
& & \beta_{2^{n}}
\end{array}\right]
$$


so that

$$
H_{n}=\left[\begin{array}{c|c}
A_{n} & \\
\hline & 1-A_{n}
\end{array}\right], N_{n}=\left[\begin{array}{c|c}
B_{n} & 0 \\
\hline C_{n} & B_{n}^{*}
\end{array}\right] .
$$

Let

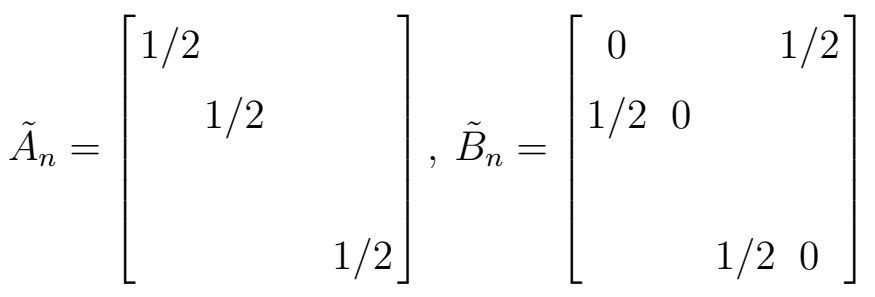

so that

$$
\tilde{H}_{n}=\left[\begin{array}{c|c}
\tilde{A}_{n} & \\
\hline & 1-\tilde{A}_{n}
\end{array}\right], \tilde{N}_{n}=\left[\begin{array}{c|c}
\tilde{B}_{n} & \\
\hline & \tilde{B}_{n}^{*}
\end{array}\right] .
$$

By Berg's technique, $[1,10]$, there exists a unitary $W \in M_{2^{n+1}}$ such that, for some constant $C$, independent of $n$,

$$
\begin{gathered}
\left\|W\left[\begin{array}{c|c}
A_{n} & \\
\hline & \tilde{A}_{n}
\end{array}\right] W^{*}-\left[\begin{array}{c|c}
A_{n} & \\
\hline & \tilde{A}_{n}
\end{array}\right]\right\| \leq C 2^{-n / 2}, \\
\left\|W\left[\begin{array}{l|l}
B_{n} & \\
\hline & \tilde{B}_{n}
\end{array}\right] W^{*}-B_{n+1}\right\| \leq C 2^{-n / 2}
\end{gathered}
$$

and (by keeping $W$ trivial except for vectors in a "segment" avoiding the "last" basis vector in each copy of $\mathbf{C}^{2^{n}}$ )

$$
W\left[\begin{array}{l|l}
0 & \\
\hline & C_{n}
\end{array}\right] W^{*}=\left[\begin{array}{l|l}
0 & \\
\hline & C_{n}
\end{array}\right] .
$$

Let $\hat{W}=W \otimes I_{2}$. It follows from above that

$$
\hat{W}\left[\begin{array}{cc|c|c}
A_{n} & & \\
& \tilde{A}_{n} & \\
& & 1-A_{n} \\
& & 1-\tilde{A}_{n}
\end{array}\right] \hat{W}^{*} \approx\left[\begin{array}{cc|c}
A_{n} & \\
& \tilde{A}_{n} & \\
\hline & 1-A_{n} \\
& 1-\tilde{A}_{n}
\end{array}\right]
$$


and

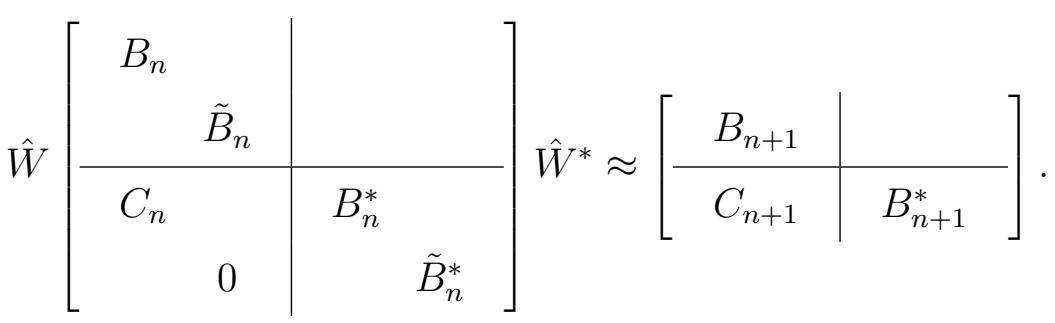

Notice that the two matrices on the left satisfy (1) By taking a path of unitaries from $W$ to $I$, we get paths satisfying (1) from $H_{n} \oplus \tilde{H}_{n}, N_{n} \oplus \tilde{N}_{n}$ to this pair. Linear interpolation from this pair to the pair on the right-hand side gives a path satisfying (1), perhaps after increasing $\epsilon_{n}$. Finally, it is a simple matter to slide the scalars which are on the diagonal of

$$
\left[\begin{array}{cc|c}
A_{n} & & \\
& \tilde{A}_{n} & \\
\hline & & 1-A_{n} \\
& & 1-\tilde{A}_{n}
\end{array}\right]
$$

to connect the right-hand pair to $H_{n+1}, N_{n+1}$. We may conclude that $\left(\varphi_{t}\right)$ exists with the properties specified in the lemma and $\varphi_{n}(h)=H_{n}$ and $\varphi_{n}(N)=N_{n}$.

\section{References}

[1] I. D. Berg, On operators which almost commute with the shift, J. Operator Theory, 11 (1984) 365-377.

[2] M.-D. Choi, Almost commuting matrices need not be nearly commuting, Proc. Amer. Math. Soc., 102 (1988), 529-533.

[3] A. Connes and N. Higson, Deformations, morphismes asymptotiques et K-theory bivariante, C. R. Acad. Sci. Parie, t. 310 Série I (1990) 101-106.

[4] M. Dădărlat and A. Nemethi, Shape theory and connective K-theory, J. Operator Theory, 23 (1990), 207-291.

[5] M. Dădărlat and T. A. Loring, in preparation.

[6] G. A. Elliott, On the classification of $C^{*}$-algebras of the real rank zero, preprint.

[7] R. Exel and T. A. Loring, Invariants of almost commuting unitaries, J. Funct. Anal., 95 (1991) 364-376.

[8] S. Klimek and A. Lesniewski, Quantum Riemann surfaces I, The unit disk, preprint.

[9] T. A. Loring, Deformations of nonorientable surfaces as torsion E-theory elements, preprint.

[10] T. A. Loring, Berg's technique for pseudo-actions with applications to AF embeddings, Canad. J. Math., 43 (1991) 119-157. 
[11] M. A. Rieffel, Deformation quantization for actions of $\mathbf{R}^{d}$, preprint.

[12] S. L. Woronowicz, Twisted SU(2) group. An example of a non-commutative differential calculus, Publ. RIMS, Kyoto Univ., 23 (1987), 117-181. 